\title{
Der Aculeatenstich.
}

Von

\section{Dr. Joseph Langer,}

gew. Externarzt an der dermatologisehen Klinik des Prof. Piok, jetzt klin. Assistent am Kaiser Franz Joseph-Kinderspitale in Prag.

I.

Der Verlauf des Bienenstiches beim normalen und bienengiftfesten Menschen.

Zwei Umstände bewogen mich im Anschluss an meine Untersuchungen über die Natur des Bienengiftes ${ }^{1}$ ) nähere Untersuchungen über die physiologischen Wirkungen dieses Giftkörpers anzustellen: einerseits vermisste ich bei Durchsicht der Literatur und der in Betracht kommenden Lehrbücher nähere Beschreibungen über den Verlauf der Hautentzündung nach Verletzungen durch Giftinsecten, andererseits hatte die unter den Bienenzüchtern schon längst bekannte Beobachtungsthatsache der allmäligen Angewöhnung des menschlichen Körpers an das Bienengift von jeher mein Interesse gefesselt und mir gerade in der Jetztzeit eine Erprobung dieser Laienbeobachtung auf ihren reellen Werth hin wünschenswerth erscheinen lassen, da die Haut als sichtbares Organ für Immunitätserscheinungen in Betracht kommt.

Ich will mich auf die Mittheilung jener Thatsachen beschränken, die ich theils durch eine ziemlich grosse eigene, mitunter experimentelle Erfahrung, theils durch Sichtung eines mittelst zweckentsprechend abgefassten Fragebögen von Bienenzüchtern erhaltenen Materiales zu sammeln Gelegenheit hatte.

Wie bekannt, verletzen die Aculeaten (Biene, Wespe, Hornis, Hummel) mit dem im Hinterleibe gelegenen und durch eine unter dem After befindliche Oeffnung hervorschnellbaren

") Langer, "Ueber das Gift unserer Honigbiene". Archiv für experimentelle Pathologie und Pharmakologie. 1897, p. ऽ81. 
Chitinstachel, dex mit eimem Giftapparate (Giftdrüse, Giftblase) in Verbindung steht.

Das Vorfinden eines Stachelapparates am Orte der Verletzung gilt als charakteristisch für den Bienenstich; dies trifft für die grosse Mehrzahl der Bienenstiche zu und ich habe in meiner obigen Arbeit auf die causalen Momente hiefür bereits hingewiesen. Immerhin kann es auch vorkommen, dass der Bienenstachel an der Stichstelle fehlt; dies ereignet sich sehr häufig bei schiefer Richtung des Stiches, wo der Stachel vorwiegend in die Epidermis applicirt wird; in solchen Fällen kann das Entzündungsbild ganz fehlen oder es pflegt sich nur rudimentär auszubilden. Längere Zeit nach dem Stiche kann selbstverständlich die durch Kratzen oder andere Abwehrbewegungen erreichte Elliminirung des zurückgelassenen Stachels eine genaue Bestimmung des Insectes verhindern. Sehr interessant ist es, den an der Stichstelle nach einem Bienenstiche zurïckbleibenden Stechapparat zu beobachten; derselbe bewegt sich beständig in Folge der Contractionen seiner Muskeln und dadurch wird einerseits der Stachel tiefer in das thierische Gewebe hineingetrieben, andererseits eine vollständige Entleerung der Giftblase bewirkt; als Ursache dieser automatischen Bewegungen müssen wir die Ausstattung des Giftapparates mit einem eigenen nervösen Centrum ansehen, welches mit herausgerissen wird und eine Zeitlang (bis 20') weiter zu leben rermag.

Die physiologische Wirkung des Bienengiftes auf den thierischen Organismus besteht im Herbeifiuhren einer localen Eutzündung, bei welcher ich durch die mikroskopische Untersuchung eine circumscripte „Necrose mit Rundzelleninfiltration, Oedem und Hyperämie" constatiren konnte. Ebenda erwähnte ich auch bereits, dass zur Errielung dieses Effectes eine cutane oder subcutane Application des Bienengiftes nothwendig sei und dass die unversehrte Haut durch den Giftstoff gar nicht, wohl aber die Schleimhäute des Auges und der Nase specifisch irritirt werden.

$\mathrm{Zwei}$ Factoren üben einen mächtigen Einfluss auf das Zustandekommen und die Entwicklung der gewöhnlichen reactiven Entzündung nach Aculeatenstichen: 
I. Die Empfindlichkeit des Individuums für den Giftstoff.

II. Die Menge des letzteren.

Bezüglich der Empfindlichkeit dem Bienengifte ge gen über vermochte ich folgende Beobachtungen zu machen: Unempfindlich für das Bienengift von jeher (auch beim Beginne der Bienenzucht) gewesen zu sein gaben unter 164 Imkern $^{1}$ ) an . . . . . . . . . . . 11 Empfindlich für das Bienengift bei Beginn der Bienenzucht waren . . . . . . . . 153 Geringer empfindlich für das Bienengift während ihrer Imkerei wurden . . . . . . . . . 126 Empfindlich für das Bieneng ift wie bei Beginn ihrer Imkerei blieben . . . . . . . . . . 27

Man ersieht daraus, dass nur ein kleiner Theil der Menschen von Natur aus für das Bienengift unempfindlich ist, während die grosse Menge einen verschiedenen Grad ron reactiver Empfindlichkeit aufweist.

Um einen richtigen Einblick in diese graduelle Empfindlichkeit zu gewinnen, ist es nothwendig, sich den gewöhnlichen Ablauf der dem Bienenstiche folgenden Entzïndung zu vergegenwärtigen. Ich habe derartige Verletzungen in einer nach Hunderten zählenden Zahl theils an mir oder an Anderen - Imkern und Nichtimkern zu beobachten Gelegenheit gehabt und ich will deshalb kurz den Verlauf eines Bienenstiches beschreiben, wie er sich vor circa 3 Jahren wiederholt in der Haut meines Vorderarmes abzuspielen pflegte.

Die mit der Pinzette oder 2 Fingern vorsichtig gefasste Biene applicirt, anf die Haut gebracht, sofort ihren Stachel und ich empfinde einen sehr intensiven, brennenden Schmerz im selben Momente, der zu Abwehrbewegungen auffordert. Um den automatisch fortarbeitenden Stachel, der an der Stichstelle zurückbleibt, während die Biene davonfliegt, bildet sich eine kleine, cutane Blutung; bereits nach 1 Minute sehe ich mehrere kleinste, miliare, blasse Efflorescenzen um den

1) Es waren gegen 600 Fragebögen versendet worden. 
Blutpunkt sich auftreten, durch deren Confluiren eine linsengrosse, blassweisse $Q$ uaddel entsteht; an der Peripherie dieser tritt zugleich ein ringförmiger Hof kleinfleckiger Röthung auf. Während nun das anfängliche Schmerzgefühl abklingt und bei der Bildung der Quaddel ein zum Kratzen aufforderndes Juckgefühl sich einstellt, wird letztere binnen 10 Minuten kreuzergross und hat nach circa 20 Min. die Grösse eines Thalers erreicht. Thre Peripherie zeigt mehrere unregelmässige blasse Zacken, welche sich in die fingerbreit lebhaft geröthete umgebende Haut erstrecken. In der Quaddel selbst prävalirt mächtiges Juckgefühl neben zeitweise blitzartig anftretendem und wieder nachlassendem brennenden Schmerz an der. Stichstelle, der zweifelsohne durch das Tieferdringen des Stachels und der Application des Giftes in neue Gewebspartien bedingt ist; nach dem Erlöschen der automatischen Stachelbewegungen tritt niemals mehr eine Schmerzempindung an der Stichstelle auf.

Allmälig schwillt die Haut in der Umgebung der Quaddel an; letztere selbst wird lebhaft roth und binnen einer Stunde nach dem Stiche findet sich die Haut in bis über Handtellergrösse entzïndlich geschwollen; diese Anschwellung pflegte sich manchmal in den nächsten Stunden noch etwas zu verbreitern, blieb aber dann auf der erlangten Höhe durch circa 20 Stunden, öfters auch noch länger bestehen; an der Stichstelle selbst vermochte ich ein kleines, derber anzufühlendes Knötchen zu tasten. (Subjectiv kann sich neben leichtem Jucken die durch die Schwellung bedingte Spannung der Haut, eventuelle Gebrauchsbehinderung der verletzten Glieder (Finger, Augenlider, Lippe, Zunge) in unangenehmer Weise geltend machen.)

Im Verlaufe des zweiten Tages tritt unter sichtlicher Abschwellung der entzündeten $\mathrm{Haut}$ das oben erwähnte Knötchen an der Stichstelle durch seine dunkelrothe Färbung und mässige Prominenz umso prägnanter hervor; durch Annahme der normalen Hantfarbe, Abschülferung seiner Epidermis, allmälige Resorption entzieht sich binnen weiteren 10-14 Tagen dieses Knötchen sowohl dem Auge als auch der Palpation und es ist deshalb unmöglich, nach längerer Zeit derartige Verletzungsstellen wieder aufzufiuden. 
Sehr häufig, namentlich wenn ein grösserer Blutpunkt sich gebildet hatte, trat an der Stichstelle ein kleines, mit eiterähnlichem Inhalte gefülltes Bläschen auf; bei der mikroskopischen Untersuchung desselben fand ich wohl reichliche Leukocyten mit aufgenommenen rothen Blutkörperchen oder deren Resten, niemals aber pflanzliche Mikroorganismen; das culturelle Verfahren ergab gleichfalls stets negative Resultate.

Wenn ich den Ablauf der Hautentzïndung nach einem Bienenstiche an meinem Vorderarme als Durchschnittsbeispiel aufstelle, so ist es nicht schwierig, in diesem acut verlaufenden Entzündungsprocesse drei zeitlich und symptomatisch wohl geschiedene Stadien zu unterscheiden:

1. Das progressive Stadium: Vom Momente des Stiches beginnend und $1 \frac{1 / 2}{2}-2$ Stunden dauernd mit folgenden Symptomen: Schmerz, Blutpunkt, Quaddelbildung, $\mathrm{Hautsch}$ wellung.

2. Das stationäre Stadium, welches dem ersten sich direct anschliesst und durch das Bild der in verschieden grosser Ausdehnung ge schwell ten Haut repräsentirt wird mit einer Dauer von $1-1 \frac{1}{2}$ Tag.

3. Das regressive Stadium; es schliesst sich direct dem zweiten an, dauert oft 8-14 Tage und ist charakterisirt durch den Rückgang der Hautschwellung, Juckgefühl, deutliches Hervortreten des Knötchens an der Stichstelle in der ersten Zeit und langsames Verschwinden desselben in weiteren Verlaufe.

Mannigfache Abweichungen von diesem Verlaufsschema werden durch die Empfindlichkeit des gestochenen Individuums ebenso herbeigeführt, wie durch die Menge des applicirten Giftes.

Die ron $\mathrm{Natur}$ aus für das Bienengift weniger oder jiberhaupt unempfindlichen Menschen ${ }^{1}$ ) erwähnen übereinstimmend, dass sie den Schmerz im Momente des Stiches empfinden und dass sich an der Stichstelle ein kleiner weisser Fleck, die Quaddel, bildet, welcher aber binnen Kurzem -

1) Die bezüglich "hereditärer Immunität" geptiogenen Erhebungen führten zu keinem verwerthbaren Resultate. 
1/. Stunde - spurlos zu verschwinden pflege; öfters constatirten sie das Auftreten einer kleinen cutanen Blutung an der Verletzungsstelle. Dieser Gruppe der „natürlich Bienengiftfesten“ steht eine andere Gruppe gegeniiber, welche man als „üb er emp find lic $h^{\text {" }}$ für das Toxin unserer Honigbiene bezeichnen muss.

Derartige Individuen - es fanden sich unter 164 Bienenzuichtern 28 - reagiren bei mächtig entwickelter örtlicher Entzündung, z. B. Anschwellen einer ganzen oberen Extremität nach Bienenstichen in die Finger mit 5-8tägiger Geschwulstdauer mit Symptomen, die den Organismus im allgemeinen und auch die Haut in diffuserer Weise betreffen.

In ersterer Richtung sind plötzliches Angst- und Schwächegefühl, Schweissausbruch, Ohnmacht, Brechneigung, Diarrhoe, Frösteln, Zittern, Unruhe, Schwindel, vermehrter Herzschlag zu nennen, und zwar treten solche Symptome oft schon nach einem einzigen Stiche auf, um bei einer Häufung von Stichen eine so mächtige Steigerung zu erfahreı, dass derartige Leute öfters zu einem mehrtägigen Krankenlager gezwungen worden zu sein angaben. $\mathrm{Ob}$ die in der medicinischen und apistischen Literatur mitgetheilten Todesfälle von Menschen nach einem oder zahlreichen Bienenstichen in einer derartigen Hyperaesthesie für dieses Toxin ihren Grund haben, lässt sich derzeit nicht feststellen. Dass die in der Regel für den menschlichen Körper ungefährliche Giftmenge einer oder einzelner Bienen in ihrem Multiplum zur dosis letalis werden kann, ist sowohl durch die Erfahrung (Tod ron Menschen und grossen Hausthieren, z. B. Pferdeu durch Ueberfallenwerden von Bienenschwärmen), als auch thierexperimentell von mir festgestellt worden.

Was die Hauterscheinungen anbelangt, so wäre das Auftreten einer Urticaria hervorzuheben.

14 Bienenzüchter beobachteten diese an sich, und zwar einzelne bereits nach einem einzigen, einzelne nach reichlicheren Bienenstichen. Einige betonen, dass die ersten Stiche zu Beginn ihres Umganges mit Bienen stets von mächtiger Urticaria begleitet waren, während sie jetztnichts derartiges mehr bemerken.

Neben der Empfindlichkeit des Individuums für das Bienengift kommt beziiglich der Entwicklung des Entzündungsbildes 
nach einem Bienenstiche auch die Menge des applicirten Giftes in Betracht. Diese ist beim einzelnen Stiche umso geringer, je schneller und vorsichtiger der an der Stichstelle zurückgelassene Stachel sammt Adnexen entfernt wird, sie wird beim Einzelstiche am grössten, wenn der Stachel ruhig belassen wird bis zum Erlöschen der oben beschriebenen automatischen Muskelbewegungen. Es ist fernerhin hervorzuheben, dass die Giftmenge der einzelnen Biene zur Zeit des gesteigerten Stoffwechsels im Hochsommer gleichfalls quantitativ vermehrt erscheint. Physiologisch-chemisches Interesse verdient die mehrfache Beobachtung, dass Stiche zur Blïthezeit des Buchweizen (Polygonum tartaricum) in Bezug auf Schmerzhaftigkeit und Hautschwellung den ersten Rang einnehmen; ebenso werden Stiche von ruhrkranken Bienen als sehr starkwirkende bezeichnet. Mit einer Häufung von Stichen wächst aus naheliegenden Gründen auch die örtliche Reaction, und sie wird umso grösser, je dichter die einzelnen Stiche applicirt wurden.

Neben den natürlich giftesten oder gering empfindlichen und den überempfindlichen findet sich die Mehrheit der Menschen in einem Zustande von mittelstarker Emp findlichkeit für das Bienengift, die sich darin äussert, dass die erwähnten allgemeinen Symptome ausbleiben und die örtlichen Entzündungserscheinungen eine gewisse Höhe erreichen, um dann in einer gewissen Zeit wieder spurlos zurückzugehen. Als ein derartiges Beispiel möchte ich den Ablauf eines Bienenstiches an mir hinstellen.

II.

\section{Wie äussert sich nun die künstlich erworbene Bienengift- immunität?}

Von den als empfindlich angegebenen 153 Personen erfuhren 126 während eines mehrjährigen Betriebes der Bienenzucht eine sichtliche Herabsetzung ihrer reactiven Empfindlichkeit.

14 von ihnen fiihlen sich derzeit giftfest; sie betonen, dass weder der einzelne Stich noch mehrere Reactionen her- 
vorrufen, abgesehen von der als Blutpunkt erscheinendeu Hämorrhagie an der Stichstelle, die wohl rorwiegend als mechanische Folge der Verletzung aufzufassen ist. D i e Schmerzempfindung "erscheint" bedeutend geringer, und es herrscht mehr ein Juckgefühl vor. Diese Leute gehören vorwiegend dem höheren Mannesalter an und treiben durch 4-50 Jahre Bienenzucht; bei 3 ron ihnen wurde Bienenzucht bereits. durch 3 Generationen betrieben und sowohl sie als auch ihre Geschwister sollen an und für sich weniger giftempindlich von Jugend auf gewesen sein, während 2 eher als überempindliche bei Beginn der Imkerei angesehen werden müssen.

21 Imker theilen mit, dass sie jetzt mit fast keiner odersehr geringer Anschwellung reagiren, und dass diese binnen wenigen Stunden vollkommen verschwinde. Sie alle beobachteten fast übereinstimmend, dass sich an der Stichstelle unter Empindung eines leichten Brennens eine bis über erbsengrosse weissliche $Q u$ ad del bilde, welche nach Minuten spurlos verschwinde; dabei schwelle die umgebende Haut manchmal etwas an, kehre aber binnen Stunden zur Norm zurück. Sie betonen ganz besonders, dass die Anschwellung sich namentlich an gewissen Hautstellen (um die Augenlider, an den Lippen, am Naseneingange, am Ohre, am Genitale) einstelle, selbst dann, wenn die Haut der Vorderarme gar nicht mehr reagire.

91 empfindliche Bienenzüchter constatirten nun im Verlaufe von einer mehrjährigen Imkerei eine deutliche Herabsetzung der Empfindlichkeit ihres Körpers fiur das Bienengift. Während sie bei Beginn der Bienenzucht wiederholt von Urticaria, Allgemeinsymptomen und mächtig entwickelter localer Entzuindung belästigt wurden, bleiben erstere jetzt ganz aus, und im Verletzungsgebiete sei jetzt eine sichtliche Abnahme der Entzündung sowohl bezüglich der Grösse als auch bezüglich der Dauer zu beobachten. Dieser Unterschied trat aber erst allmälig ein und es bedurfte hiezu eines mehrjährigen Zeitraumes, während dessen reichliche Bienenstiche applicirt worden waren.

Beifolgende Tabelle gewährt einen Einblick in die diesbezuiglich gemachten Beobachtungen von Bienenzüchtern: 

trat ein

Herabsetzung der Empfindlichkeit für das Bienengift bei 71 Imkern nach $1-5$ jähriger Bienenzucht

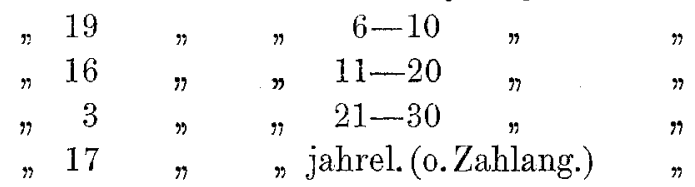

Dass während derartiger Zeiträume sehr viele Stiche erhalten werden, wird uns erst klar, wenn durchaus verlässliche Männer, Koryphäen der Bienenzucht, mittheilen, dass sie im Verlaufe der jährlichen Bienenflugzeit (März bis September) von 200 bis über 1000 Bienen gestochen wurden. Bei derartigen Berufsimkern kommt es gar nicht so selten vor, dass sie an einem Tage während ihrer Thätigkeit am Bienenstande (z. B. beim Schwarmeinfangen, Ueberlegen von Völkern etc.) von 20-100 Bienen gestochen werden und dass trotzdem nur geringe reactive Erscheinungen zur Entwicklung kommen; es ist nun ganz interessant hiebei zu erfahren, dass einzelne Bienenzüchter ihre derzeitige geringe Empfindlichkeit für das Bienengift auf das einmalige Erleiden gehäufter Stichverletzungen (30-100 Stiche) zurückführen.

Neben der Gruppe des natürlich und künstlich bienengiftfesten Menschen findet sich in meiner obigen Zusammenstellung noch eine andere, welche 27 Leute unfasst, die trotz jahrelanger Imkerei immer noch gleich empfindlich sind wie anfangs. Es muss dahingestellt bleiben, $a b$ es sich da um Individuen handelt, denen die Fähigkeit immun zu werden ganz abgeht, oder ob sich bei ihnen Immunität im Laufe der Zeit noch einstellen wird. Ich vermuthe letzteres, zumal ein hoher Percentsatz ron ihnen noch im ersten Decennium imkert.

Als Charaktere der künstlich erworbenen Bienengiftimmunität hebe ich hervor:

1. Wegbleiben der Urticaria und der Allgemeinsymptome nach selbst zahlreichen Bienenstichen bei hiefür sonst empfindlichen Individuen.

2. Veränderungen im örtlichen Entzündungsbilde, welche sowohl die Dauer, als auch die Grösse desselben betreffen: es findet eine temporäre und 
quantitativeVerringerung deroben beschriebenen drei Stadien statt.

Ein höherer Immunitätsgrad zeigt den Ausfall des stationären zweiten Stadiums, der Hautschwellung, so dass bei derartigen Leuten dem ersten Stadium direct das dritte folgt.

In der nur partiellen Entwicklung des ersten Stadiums (Schmerz, Blutung) mit abgekürztem Verlaufe des dritten (kleines Knötchen, welches binnen einigen Tagen verschwindet) erreicht die Imm unisirbarkeit des Menschen gegenüber dem Bienengifte nach meinen Beobachtungen ihren Höh epunkt.

Diese "erworbene Immunität" kann nun leicht herabgesetzt, ja getilgt werden.

So heben mehrere Bienenzüchter hervor, dass sie nach den ersten Stichen im Frihjahre auffallend stark reagiren, während sie durch diese Reimmunisirung allmälig unempfindlich selbst für Stichhäufungen zu werden pflegen.

Nach jahrelangem Aussetzen der Imkerei soll die Fmpfindlichkeit für das Bienengift oft gerade so stark wie beim Beginne sein; ebenso sollen Erkrankungen oft plötzlich einen totalen Verlust dieser durch Jahre erworbenen Eigenschaft herbeiführen können.

Für diese makroskopischen Beobachtungen dürften die im Gange befindlichen histologischen Untersuchungen des Aculeatenstiches beim normalen und künstlich giftfest gemachten Thiere interessante Details zu Tage fördern, über die ich nach Abschluss meiner diesbezïglichen thierexperimentellen Versuche berichten werde. 